\title{
General splicing factor SF2/ASF promotes alternative splicing by binding to an exonic splicing enhancer
}

\author{
Qiang Sun, ${ }^{1}$ Akila Mayeda, ${ }^{2}$ Robert K. Hampson, ${ }^{1}$ Adrian R. Krainer, ${ }^{2}$ and Fritz M. Rottman ${ }^{1,3}$ \\ Department of Molecular Biology and Microbiology, Case Western Reserve University, School of Medicine, Cleveland, Ohio \\ 44106-4960 USA; ${ }^{1}$ Cold Spring Harbor Laboratory, Cold Spring Harbor, New York 11724-2208 USA ${ }^{2}$
}

The general splicing factor SF2/ASF binds in a sequence-specific manner to a purine-rich exonic splicing enhancer (ESE) in the last exon of bovine growth hormone (bGH) pre-mRNA. More importantly, SF2/ASF stimulates in vitro splicing of bGH intron D through specific interaction with the ESE sequences. However, another general splicing factor, SC35, does not bind the ESE sequences and has no effect on bGH intron D splicing. Thus, one possible function of SF2/ASF in alternative and, perhaps, constitutive pre-mRNA splicing is to recognize ESE sequences. The stimulation of bGH intron D splicing by SF2/ASF is counteracted by the addition of hnRNP A1. The relative levels of SF2/ASF and hnRNP A1 influence the efficiency of bGH intron $D$ splicing in vitro and may be the underlying mechanism of this alternative pre-mRNA processing event in vivo.

[Key Words: Alternative splicing; bovine growth hormone pre-mRNA; SF2/ASF; hnRNP A1; exonic splicing enhancer]

Received September 3, 1993; accepted October 6, 1993.

Pre-mRNA splicing requires accurate selection of $5^{\prime}$ and $3^{\prime}$ splice sites. For many genes, alternative splice sites can be selected from a single primary transcript to generate two or more mRNAs encoding multiple protein isoforms. Although alternative pre-mRNA splicing provides an important route for the regulation of gene expression, the underlying regulatory mechanisms remain largely uncharacterized.

A central question in constitutive and alternative premRNA splicing concerns the selection of specific splice sites. Introns are defined by the presence of a conserved $5^{\prime}$ splice site, a 3 ' splice site, a polypyrimidine tract, and a less well-conserved branch site. Although these signals are required for splicing, they are insufficient to specify all introns. Several studies have shown that exon sequences can also play an important role in splice site selection (Reed and Maniatis 1986; Mardon et al. 1987; Helfman et al. 1988; Cooper and Ordahl 1989; Hampson et al. 1989; Kakizuka et al. 1990; Katz and Skalka 1990; Libri et al. 1991; Nagoshi and Baker 1990; Hedley and Maniatis 1991; Hoshijima et al. 1991; Cooper 1992; Cote et al. 1992; Watakabe et al. 1993; Xu et al. 1993). Although the precise role of these exon sequences in splicing remains unclear, recent studies on the Drosophila sex determination pathway suggest a possible function for such sequences. Alternative splicing of double-sex $(d s x)$ pre-mRNA involves utilization of two mutually ex-

${ }^{3}$ Corresponding author. clusive exons. Sequences in exon 4 regulate its inclusion positively in female-specific dsx mRNA (Nagoshi and Baker 1990; Hedley and Maniatis 1991; Hoshijima et al. 1991). The trans-acting factors encoded by the transformer (tra) and transformer 2 (tra2) genes together activate the 3 ' splice site upstream of the $d s x$ female-specific exon 4 through interaction with six 13-nucleotide repeats present in the exon (Tian and Maniatis 1992). Thus, protein factors are capable of binding to exon sequences in a sequence-specific manner and affecting splicing of introns located some distance away from the protein-binding sites.

A number of factors required for constitutive splicing have been shown to be involved in alternative splice site selection. The product of the Sex-lethal (SxI) gene regulates alternative splicing of tra pre-mRNA by repressing the inclusion of exon 2 (Boggs et al. 1987). Recent results showed that Sxl inhibits the use of the $3^{\prime}$ splice site upstream of exon 2 by blocking the binding of the essential splicing factor U2AF to the polypyrimidine tract (Valcarcel et al. 1993). Binding of U1 small nuclear ribonucleoprotein (snRNP) to a pseudo-5' splice site, as opposed to the authentic $5^{\prime}$ splice site, has been implicated in the regulated alternative intron retention of Drosophila P-element pre-mRNA in somatic cells /Siebel et al. 1992). Both U2AF and U1 snRNPs have been implicated in exon definition interactions that specify splicing of alternative exon 4 of preprotachykinin (Hoffman and Grabowski 1992). 
Two protein factors, SF2/ASF and heterogeneous ribonucleoprotein (hnRNP) A1, counteract each other in a concentration-dependent manner to define alternative $5^{\prime}$ splice site selection, as well as exon skipping and inclusion, of a variety of pre-mRNAs in vitro /Ge and Manley 1990; Krainer et al. 1990b; Mayeda and Krainer 1992; Mayeda et al. 1993). SF2/ASF, as well as SC35, can also influence alternative $3^{\prime}$ splice site selection in vitro (Fu et al. 1992). Both SF2/ASF and SC35 belong to the SR (Ser- and Arg-rich) protein family, a group of highly conserved nuclear phosphoproteins (Fu et al. 1992; Mayeda et al. 1992; Zahler et al. 1992). SR proteins appear to have redundant or overlapping, but in some cases, distinct, functions in constitutive and alternative pre-mRNA splicing (Fu et al. 1992; Kim et al. 1992; Mayeda et al. 1992; Zahler et al. 1992). These studies suggest that the relative amounts of SR proteins in different cell or tissue types may help to determine splice site selection. The precise molecular mechanisms by which these factors participate in constitutive and alternative splicing remain to be characterized.

The last intron (intron D) of bovine growth hormone (bGH) pre-mRNA is alternatively spliced in the pituitary, where a small fraction of the cytosolic bGH mRNA retains intron D (Hampson and Rottman 1987). The identical pattern of alternative intron retention has also been conserved in human growth hormone premRNA (Cooke et al. 1988). Splicing of bGH intron D is dependent on the downstream 115-nucleotide FP element, a purine-rich exonic splicing enhancer (ESE), both in transfected cells and in vitro (Hampson et al. 1989; Sun et al. 1993). A competition assay revealed that the influence of the FP element on splicing of the upstream bGH intron $D$ is mediated through interaction with one or more trans-acting factors. A UV cross-linking assay identified a $35-\mathrm{kD}$ protein factor that binds specifically to the FP element (Sun et al. 1993). In the work reported here, we have characterized this $35-\mathrm{kD}$ protein factor and found it to be the general splicing factor SF2/ASF. SF2/ASF promotes splicing of bGH intron D through specific interaction with the FP element in bGH terminal exon 5. SC35, which has equivalent in vitro splicing activities, has no effect on bGH intron D splicing. Furthermore, the positive influence of SF2/ASF on the splicing of bGH intron D can be counteracted by hnRNP A1 protein.

\section{Results}

The 35-kD protein factor that binds specifically to the FP element is absent in HeLa cell $\$ 100$ extracts

Previous work showed that a $35-\mathrm{kD}$ protein factor(s) binds specifically to the FP element in bGH terminal exon 5 (Sun et al. 1993). To further characterize this putative $35-\mathrm{kD}$ protein factor(s), two RNA substrates were made. E5/FP RNA is a 127-nucleotide T7 RNA polymerase runoff transcript containing the 115-nucleotide FP element and 12 additional nucleotides of vector sequence. E5/FPD RNA is a 147-nucleotide T7 transcript that lacks the FP element and contains instead a 135nucleotide sequence of bGH exon 5 plus the same 12nucleotide vector sequence present in E5/FP RNA (Fig. 1A). The E5/FP RNA employed in these experiments is identical to the FP1 RNA used in previous studies (Sun et al. 1993) except that the E5/FP RNA lacks 47 nucleotides of vector sequence. When radiolabeled E5/FP RNA was UV cross-linked in HeLa cell nuclear extract, both in the presence and absence of ATP and $\mathrm{Mg}^{2+}$, two major cross-linked bands of 55 and $35 \mathrm{kD}$ were observed (Fig. 1B, lanes 3,4). The appearance of both cross-linked bands was dependent on addition of nuclear extract (Fig.

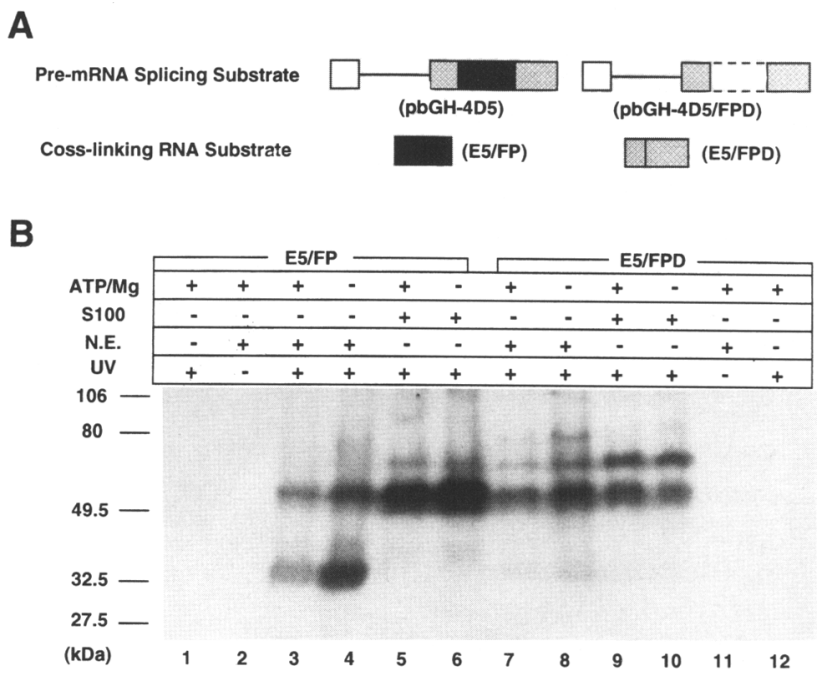

C

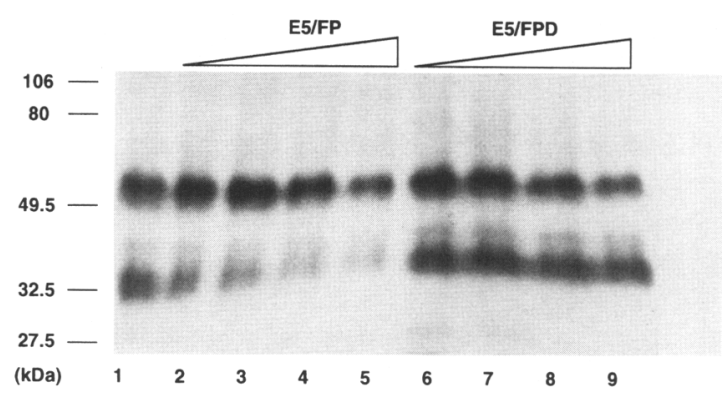

Figure 1. Characterization of the $35-\mathrm{kD}$ protein factor(s). $(A)$ RNA substrates used for in vitro splicing reactions and UV cross-linking assays. Solid areas denote the FP element; lightly shaded areas denote other exon 5 sequences excluding the FP element. (B) UV cross-linking of ${ }^{32}$ P-labeled E5/FP and E5/FPD RNA in HeLa cell nuclear extract (N.E.) or S100 extract (S100). UV irradiation, additions of ATP and $\mathrm{Mg}^{2+}(\mathrm{ATP} / \mathrm{Mg})$, N.E., and S100 are indicated by plus $(+)$ and minus $(-)$ signs. The crosslinked material was analyzed by SDS-PAGE after RNase A treatment, as described in Materials and methods. $(C)$ Crosslinking of the $35-\mathrm{kD}$ protein(s) to E5/FP RNA in nuclear extract is competed by increasing amounts of unlabeled E5/FP RNA, but not by E5/FPD RNA. (Lane 1) no competitor was added. Molar excess (4-, 7.5-, 15-, and 30-fold) of unlabeled E5/FP RNA (lanes 2-5) or unlabeled E5/FPD RNA (lanes 6-9) was added at the same time as the labeled E5/FP RNA. Protein standards are low-range prestained markers (Bio-Rad). 
1B, lane 1) and UV irradiation (Fig. 1B, lane 2). When radiolabeled E5/FP RNA was cross-linked in HeLa cell S100 extract, however, only the $55-\mathrm{kD}$ and a new $65-\mathrm{kD}$ cross-linked band were observed, both in the presence and absence of ATP and $\mathrm{Mg}^{2+}$ (Fig. 1B, lanes 5,6). Thus, the cross-linked $35-\mathrm{kD}$ polypeptide present in nuclear extract appears to be absent from S100 extract.

To confirm the binding specificity of this $35 \cdot \mathrm{kD}$ protein/s) to the FP sequence in bGH exon 5, RNA lacking the FP element (E5/FPD) was used in UV cross-linking assays. We hypothesized that if the binding of this $35-\mathrm{kD}$ protein(s) is specific, the protein should only recognize E5/FP RNA, but not E5/FPD RNA, because exon 5 lacking the FP element is unable to activate splicing of the upstream bGH intron D. When radiolabeled E5/FPD RNA was cross-linked in HeLa cell nuclear extract, either in the presence or absence of ATP and $\mathrm{Mg}^{2+}$, only a trace of $35-\mathrm{kD}$ cross-linked protein could be detected (Fig. 1B, lanes 7,8), further suggesting that binding of the $35-\mathrm{kD}$ protein $(\mathrm{s})$ to the $\mathrm{FP}$ element is sequence specific. Binding of the 35-kD protein(s) to E5/FPD RNA was not detected in HeLa cell S100 extract under similar conditions (Fig. 1B, lanes 9,10). The 55-kD protein, which was previously shown to bind nonspecifically, cross-linked to E5/FPD RNA under all conditions tested (Fig. 1B, lanes 7-10|. Finally, the cross-linking of the $35-\mathrm{kD}$ protein(s) to E5/FP RNA was competed by increasing amounts of unlabeled E5/FP RNA (Fig. 1C, lanes 2-5), but not by unlabeled E5/FPD RNA (Fig. 1C, lanes 6-9). The observed selectivity ratio between these RNAs was greater than 10 . Therefore the $35-\mathrm{kD}$ polypeptide(s), but not the $55-\mathrm{kD}$ polypeptide(s), binds specifically to the FP element in bGH terminal exon 5 .

\section{The general splicing factor SF2/ASF binds specifically to the FP element}

The absence in HeLa cell $\mathrm{S} 100$ extracts of the $35-\mathrm{kD}$ protein factor(s) that binds specifically to the FP element suggests that it might be one of the SR proteins. These proteins are part of a conserved family of splicing factors with related biochemical activities, which are present in trace amount in S100 extracts (Fu et al. 1992; Kim et al. 1992; Mayeda et al. 1992; Zahler et al. 1992, 1993). SF2/ ASF, a member of the SR family, migrates as a doublet of $\sim 33 \mathrm{kD}$ on SDS-PAGE and is required for pre-spliceosome complex formation (Krainer et al. 1990a). Deletion of the FP element, the binding site for the putative 35-kD protein factor(s), from the bGH terminal exon 5, blocks splicing at an early step, that is, at or before pre-spliceosome complex assembly (Sun et al. 1993). A UV crosslinking/immunoprecipitation assay was performed to determine whether the $35-\mathrm{kD}$ protein $(\mathrm{s})$ that cross-links specifically to the FP element is SF2/ASF. Anti-SF2/ASF monoclonal antibody $(\mathrm{mAb})$ specifically precipitated a labeled $35-\mathrm{kD}$ protein when the E5/FP RNA was crosslinked in HeLa cell nuclear extract, either in the presence or absence of ATP and $\mathrm{Mg}^{2+}$ (Fig. 2A, lanes 2,3). The labeled $55-\mathrm{kD}$ protein(s), which binds nonspecifically to E5/FP RNA, was not precipitated by anti-SF2/
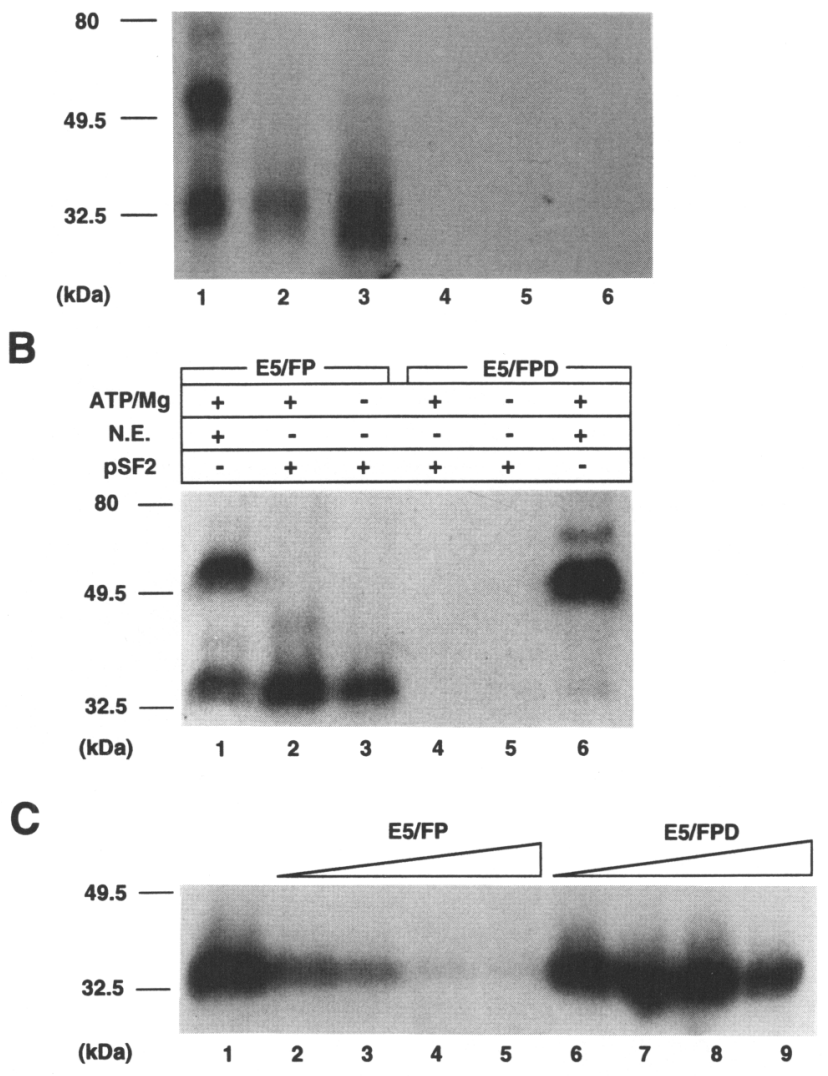

Figure 2. SF2/ASF binds specifically to the FP element. $(A)$ Immunoprecipitation of a $35-\mathrm{kD}$ protein bound specifically to the FP element with anti-SF2/ASF mAb. (Lane 1) Cross-linking of E5/FP RNA in HeLa cell nuclear extract (N.E.) without immunoprecipitation; (lanes 2-6) cross-linking of RNA and protein followed by immunoprecipitation with monoclonal antibodies as described in Materials and methods. Additions of ATP and $\mathrm{Mg}^{2+}$ (ATP/Mg), N.E., S100, and monoclonal antibodies (Ab) specific for SF2/ASF ( $\alpha$-SF2) or a control bacterial protein (Cont) are indicated by plus ( + ) and minus $(-\mid$ signs. (B) Purified SF2/ASF cross-links to E5/FP RNA, but not to E5/FPD RNA $1+$ and -1 Additions of ATP/Mg, N.E., and $0.4 \mu \mathrm{g}$ of purified SF2/ASF protein (pSF2). (C) Cross-linking of SF2/ASF to E5/FP RNA is competed by increasing amounts of unlabeled E5/FP RNA, but not by E5/FPD RNA. Each reaction contained $0.4 \mu \mathrm{g}$ of purified SF2/ASF protein and $7 \mu \mathrm{g}$ of BSA. (Lane 1) No competitor was added. Unlabeled E5/FP RNA (4-, 7.5-, 15-, and 30fold molar excess) (lanes 2-5) or unlabeled E5/FPD RNA (lanes $6-9 \mid$ was added at the same time as the labeled E5/FP RNA. Protein standards are as in Fig. 1.

ASF $\mathrm{mAb}$. The labeled $35-\mathrm{kD}$ protein(s) was not precipitated by a control monoclonal antibody (Fig. 2A, lane 5), nor was it detected in anti-SF2/ASF mAb immunoprecipitates when E5/FP RNA was cross-linked in HeLa cell S100 extracts (Fig. 2A, lane 4), or when E5/FPD RNA 
was cross-linked in HeLa cell nuclear extracts (Fig. 2A, lane 6). Because anti-SF2/ASF mAb did not quantitatively precipitate either the cross-linked $35-\mathrm{kD}$ band or purified SF2/ASF protein (data not shown), we could not address whether SF2/ASF is the only $35-\mathrm{kD}$ protein that specifically binds to the FP element in nuclear extracts.

Cross-linking of a $35-\mathrm{kD}$ protein(s) to E5/FP RNA, but not to E5/FPD RNA, was observed in HeLa cell S100 extracts complemented with purified human SF2/ASF protein (data not shown). This observation is consistent with SF2/ASF being the 35-kD E5/FP RNA-binding protein that is present in trace amounts in $\mathrm{S} 100$ extracts. We then examined whether specific binding to the FP element is an intrinsic property of SF2/ASF or whether interactions with other components are required. UV cross-linking experiments were carried out with purified HeLa cell SF2/ASF, in the absence of S100 extract. E5/FP RNA cross-linked to purified SF2/ASF, either in the presence or absence of ATP and $\mathrm{Mg}^{2+}$ (Fig. 2B, lanes 2,3). The electrophoretic mobilities of the $35-\mathrm{kD}$ protein in nuclear extract and purified SF2/ASF after cross-linking to E5/FP RNA were identical (Fig. 2B, cf. lanes 1-3). E5/FPD RNA, however, did not cross-link to purified SF2/ASF under similar conditions (Fig. 2B, lanes 4,5). The cross-linking of SF2/ASF to E5/FP RNA could be competed by increasing amounts of unlabeled E5/FP RNA (Fig. 2C, lanes 1-5), and only slightly by E5/FPD RNA (Fig. 2C, lanes 6-9), further indicating that SF2/ ASF is capable of specifically recognizing the FP element in the absence of other factors. The observed selectivity ratio of purified SF2/ASF for E5/FP versus E5/FPD RNA was $>10$, similar to that observed for the $35-\mathrm{kD}$ protein in nuclear extract.

\section{SF2/ASF promotes splicing of $b G H$ intron $D$ through specific interaction with the FP element in the downstream exon}

It is important to determine whether SF2/ASF binding to the FP element is functionally significant, that is, capable of activating the splicing of upstream intron $\mathrm{D}$. When the wild-type bGH pre-mRNA substrate, pbGH-4D5 (Fig. 1A), was spliced in HeLa cell nuclear extract, addition of purified SF2/ASF stimulated the splicing of bGH intron D (Fig. 3A, cf. lanes 1 and 2). Splicing of bGH intron $\mathrm{D}$ was inhibited by a 10 -fold molar excess of unlabeled E5/FP specific competitor RNA (Fig. 3A, lane 3) but not by a 100-fold molar excess of E5/FPD nonspecific competitor RNA (Fig. 3A, lane 4). When both specific competitor (E5/FP RNA) and purified SF2/ASF were added to the same reaction, SF2/ASF relieved the inhibition of splicing (Fig. 3A, lane 5, cf. lane 3), presumably by compensating for the loss of endogenous SF2/ASF titrated by the added E5/FP RNA. The stimulation of bGH intron D splicing by SF2/ASF is $\sim 5$ - to 10-fold, which is comparable to the SF2/ASF selectivity ratio in the binding assays. This result suggests that the SF2/ASF binding to the FP element has functional significance and is responsible for activation of intron $\mathrm{D}$ splicing. Addition of SF2/ASF to reactions containing the mutant bGH pre-
A

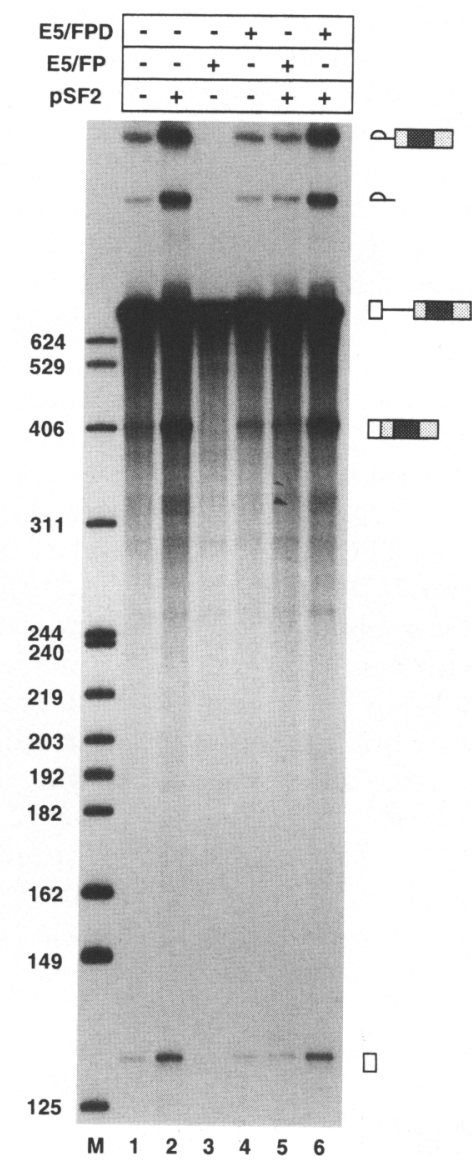

B

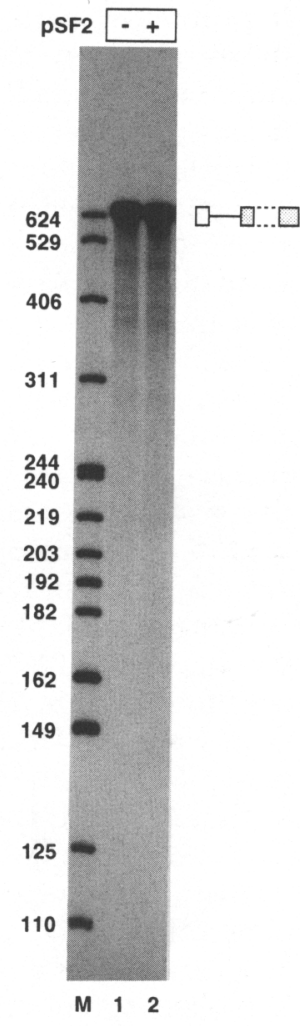

Figure 3. SF2/ASF promotes splicing of bGH intron D through specific interaction with the FP element. $|A|$ SF2/ASF stimulates splicing of bGH intron D. Pre-mRNA substrate pbGH-4D5 was spliced in nuclear extract for $90 \mathrm{~min} .(+$ and -) Additions of 10-fold molar excess of E5/FP RNA, 100-fold molar excess of E5/FPD RNA, and $0.4 \mu \mathrm{g}$ of purified human SF2/ASF protein (pSF2). (B) SF2/ASF fails to activate splicing of bGH mutant pre-mRNA lacking the FP element. Pre-mRNA substrate pbGH-4D5/FPD was incubated under splicing conditions in nuclear extract for $90 \mathrm{~min}$ in the absence (lane 1) or the presence (lane 2) of $0.4 \mu \mathrm{g}$ of purified human SF2/ASF (pSF2). Pre-mRNA, spliced products, and intermediates are indicated by diagrams. The molecular size markers (lane $M$ ) are pBR322 digested with HpaII.

mRNA substrate pbGH-4D5/FPD (Fig. 1A), however, had no effect on splicing of bGH intron D (Fig. 3B, lanes 1,2 ), that is, splicing was undetectable regardless of the addition of SF2/ASF. Therefore, the FP element in the downstream exon, which interacts specifically with SF2/ ASF, is required for activation of intron $D$ splicing by SF2/ASF.

SC35 cannot be not cross-linked to the FP element in the downstream exon and does not stimulate $b G H$ intron $D$ splicing

The SF2/ASF protein used in the experiments described 
earlier was a highly purified preparation from HeLa cells. To rule out the possibility that specific binding and splicing activation are attributable to trace contaminating proteins in the SF2/ASF preparation, the experiments were repeated with purified recombinant SF2/ ASF (Càceres and Krainer 1993). Recombinant SF2/ASF has a somewhat lower specific activity than HeLa cell SF2/ASF as shown by complementation of S100 extract for splicing of adenovirus and $\beta$-globin pre-mRNA substrates (data not shown). This is probably attributable to the required renaturation procedure and/or to the lack of post-translational modifications /Càceres and Krainer 1993). When recombinant SF2/ASF was used in the UV cross-linking assay, it bound to E5/FP RNA in the presence or absence of ATP and $\mathrm{Mg}^{2+}$ (Fig. 4A, lanes 6,7) and much more weakly to E5/FPD RNA under similar conditions (Fig. 4A, lanes 8,9). When wild-type bGH premRNA (pbGH-4D5) was spliced in HeLa cell nuclear extract, addition of recombinant SF2/ASF also stimulated splicing of bGH intron D (Fig. 4B, cf. lanes 1 and 4). Thus, recombinant SF2/ASF exhibited similar RNA-binding specificity and bGH splicing stimulatory activity as the highly purified HeLa cell SF2/ASF, confirming that SF2/ ASF promotes splicing of $b G H$ intron $D$ through specific interaction with the downstream exonic FP element.

SC35, another SR protein of similar size, has been reported to have very similar in vitro splicing activities to those of SF2/ASF (Fu et al. 1992). We therefore tested whether SC35 can also activate bGH intron D splicing. When recombinant SC35 was used in the UV cross-linking assay, it cross-linked neither to E5/FP RNA nor to E5/FPD RNA (Fig. 4A, lanes 2-5). When wild-type bGH pre-mRNA was spliced in HeLa cell nuclear extract, addition of recombinant SC35 had no effect on splicing of bGH intron D (Fig. 4B, cf. lanes 1 and 3). However, the same preparation of recombinant SC35 had higher general splicing activity than recombinant SF2/ASF when identical amounts of both recombinant proteins were tested in the $\$ 100$ complementation assay with an adenovirus pre-mRNA substrate (data not shown) and a $\beta$-globin pre-mRNA substrate (Fu et al. 1992). Therefore, in contrast to SF2/ASF, SC35 does not interact specifically with the FP element and does not promote splicing of bGH intron $\mathrm{D}$.

\section{hnRNP A1 counteracts the activation of $b G H$ intron $D$ splicing by $S F 2 / A S F$}

Heterogeneous ribonucleoprotein (hnRNP) Al has been shown to counteract the effect of SF2/ASF in alternative splicing events involving $5^{\prime}$ splice site selection and exon skipping or inclusion, but not $3^{\prime}$ splice site selection (Fu et al. 1992; Mayeda and Krainer 1992; Mayeda et al. 1993). It was of interest to determine whether hnRNP $\mathrm{A} 1$ also counteracts SF2/ASF in alternative intron retention. Addition of recombinant hnRNP Al protein to the in vitro splicing reaction inhibited splicing of bGH intron D (Fig. 5, cf. lanes 1 and 2). Moreover, further addition of SF2/ASF reversed the inhibitory effect of hnRNP $\mathrm{Al}$ and restored splicing of bGH intron $\mathrm{D}$ (Fig. 5, cf. lanes 2-4). As shown above, SF2/ASF relieved the inhibition of bGH intron $\mathrm{D}$ splicing by the specific competitor $\mathrm{E} 5 / \mathrm{FP}$ RNA (Fig. 3A, lanes 3,5; Fig. 5, lanes 5,9). However, addition of either hnRNP Al alone or a combination of SF2/ASF and hnRNP Al failed to relieve the inhibition of bGH intron D splicing by E5/FP RNA (Fig. 5, lanes $7,8)$. In the presence of the nonspecific competitor E5/ FPD RNA, the effect of hnRNP Al was similar to that without addition of any competitor RNA (Fig. 5, cf. lanes $2-4$ and 10-12). Thus, hnRNP Al counteracts the stim-
Figure 4. SC35 does not bind to the FP element or stimulate splicing of bGH intron D. (A) Recombinant SF2/ASF, but not recombinant $\mathrm{SC} 35$, protein binds specifically to E5/FP RNA. (+ and -) Additions of ATP/Mg, HeLa cell nuclear extract (N.E.), E5/FP or E5/FPD RNAs, $1.3 \mu \mathrm{g}$ of recombinant SF2/ASF(rSF2) and $0.3 \mu \mathrm{g}$ of recombinant SC35 (rSC35). Protein standards are as in Fig. 1. (B) Recombinant SF2/ ASF, but not recombinant SC35, activates splicing of bGH intron D. Pre-mRNA substrate pbGH-4D5 was incubated under splicing conditions in nuclear extract for 90 min in the absence (lane 1) or the presence of $0.6 \mu \mathrm{g}$ of purified human SF2/ASF (pSF2, lane 2), $0.4 \mu \mathrm{g}$ of recombinant SC35 (rSC35, lane 3), and $2 \mu \mathrm{g}$ of recombinant SF2/ASF (rSF2, lane 4). Pre-mRNA, spliced product, and intermediates are indicated by diagrams. The molecular size markers (lane $M$ ) are pBR322 digested by $\mathrm{HpaII}$.
A

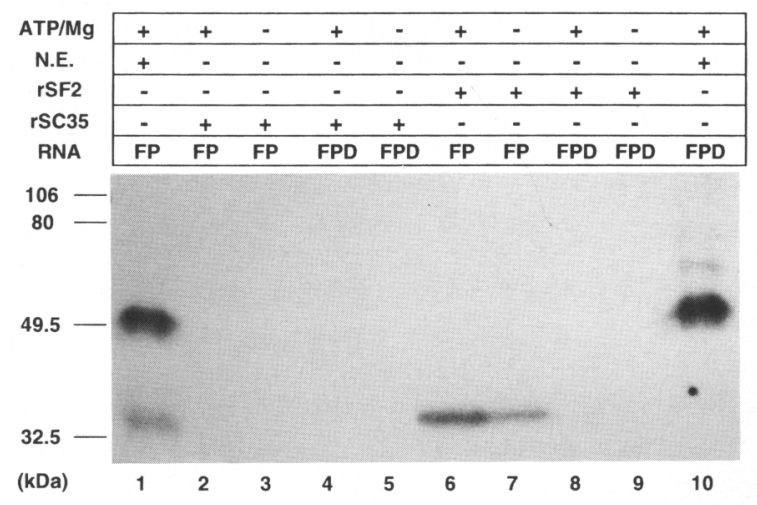

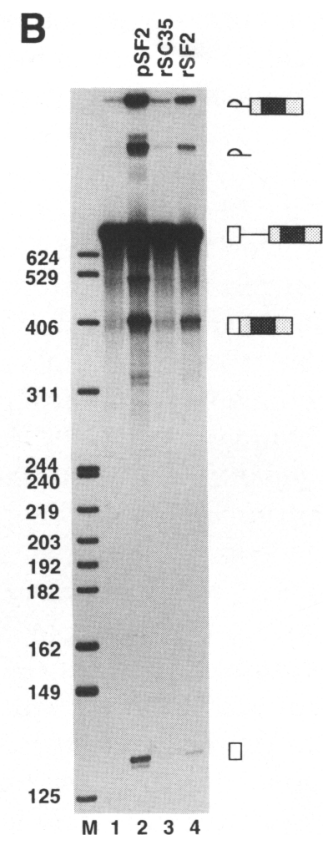




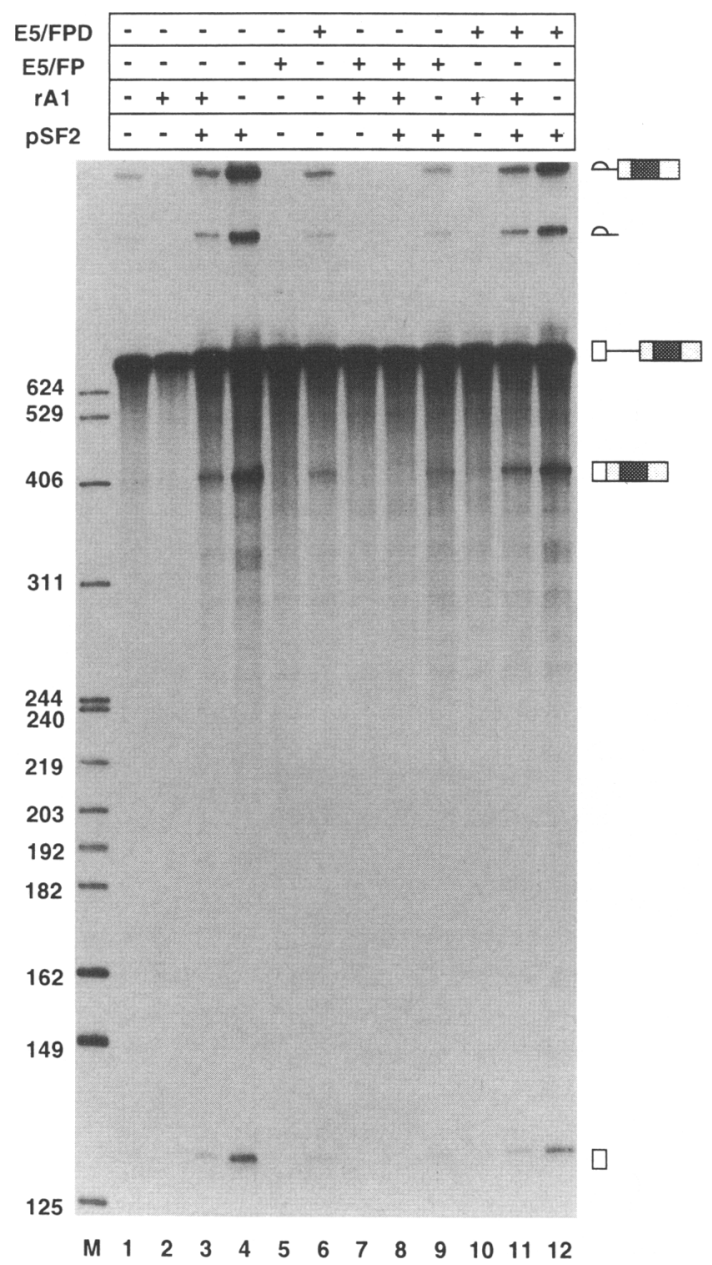

Figure 5. hnRNP Al counteracts the positive effect of SF2/ ASF on splicing of bGH intron D. Pre-mRNA substrate pbGH4D5 was spliced in HeLa cell nuclear extract for $90 \mathrm{~min} .1+$ and -) Additions of 10-fold molar excess of E5/FP RNA, 100-fold molar excess of E5/FPD RNA, $0.8 \mu \mathrm{g}$ of recombinant hnRNP A1 (rA1), and $0.4 \mu \mathrm{g}$ of purified SF2/ASF (pSF2). Pre-mRNA, spliced products, and intermediates are indicated by diagrams. The molecular size markers (lane $M$ ) are pBR322 digested with HpaII.

ulatory effect of SF2/ASF on splicing of bGH intron D. hnRNP Al does not have a general splicing inhibitory activity in the amounts used here, as it had little effect on splicing of control adenovirus (data not shown) and $\beta$-globin (Mayeda and Krainer 1992) pre-mRNAs. Moreover, comparable amounts of the same preparation switched alternative 5 ' splice site utilization without reducing overall splicing (Mayeda and Krainer 1992).

\section{hnRNP A1 binds with low specificity to the FP element}

Because hnRNP Al counteracts the stimulatory activity of SF2/ASF on splicing of bGH intron D, it was of interest to determine whether hnRNP Al also specifically recognizes the FP element. Anti-hnRNP Al mAb immunoprecipitated a labeled $35-\mathrm{kD}$ protein when E5/FP RNA was cross-linked in nuclear extract (Fig. 6A, lanes 3,4). This $35-\mathrm{kD}$ protein was not immunoprecipitated in nuclear extract by a control monoclonal antibody (Fig. 6A, lane 7). UV cross-linking of E5/FP RNA in HeLa cell S100 extract, followed by anti-hnRNP A1 monoclonal antibody immunoprecipitation yielded only a small

\section{A}

\begin{tabular}{|c|c|c|c|c|c|c|c|}
\hline \multirow[b]{2}{*}{ ATP/Mg } & & & \multicolumn{3}{|l|}{ E5/FP } & E5/FPD & \multirow{2}{*}{$\frac{\text { E5/FP }}{+}$} \\
\hline & + & + & + & - & + & + & \\
\hline$S 100$ & $=$ & + & - & - & + & - & - \\
\hline N.E. & + & - & + & + & - & + & + \\
\hline $\mathbf{A b}$ & - & - & $\alpha-\mathbf{A 1}$ & $\alpha-\mathbf{A} 1$ & $\alpha-A 1$ & $\alpha-A 1$ & Cont \\
\hline
\end{tabular}

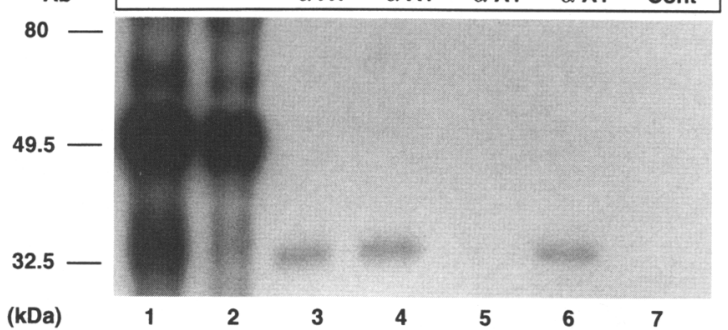

B

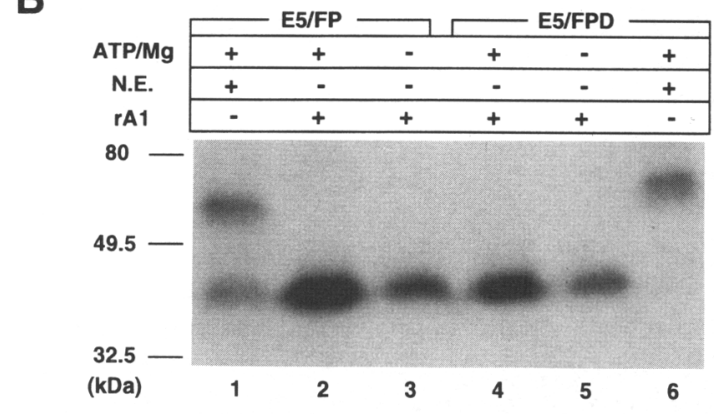

C

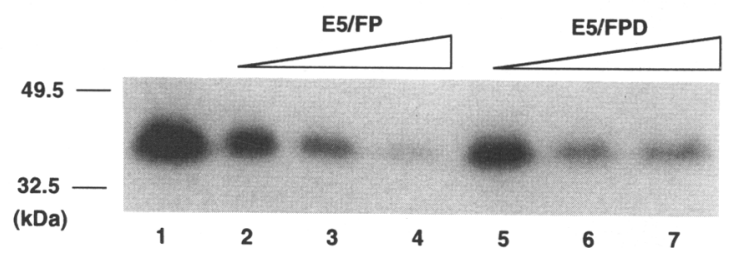

Figure 6. hnRNP Al binds with low specificity to the FP element. $(A)$ Immunoprecipitation of a $35-\mathrm{kD}$ protein that binds both E5/FP and E5/FPD RNA with anti-hnRNP Al mAb. (Lanes 1,2) UV cross-linking of E5/FP RNA in HeLa cell nuclear extract (N.E.) and S100 extract (S100) without immunoprecipitation; (lanes 3-7) cross-linking of RNA and protein followed by immunoprecipitation with monoclonal antibodies as described in Materials and methods. (+ and -) Additions of ATP/Mg, N.E., S100, and monoclonal antibodies (Ab) specific for hnRNP $\mathrm{Al}(\alpha-\mathrm{A} 1)$ or a bacterial protein (Cont). (B) Recombinant hnRNP Al protein binds to both E5/FP and E5/FPD RNA. (+ and -1 Additions of ATP $/ \mathrm{Mg}$, N.E., and $0.4 \mu \mathrm{g}$ of recombinant hnRNP Al (rAl). (C) Cross-linking of hnRNP Al to E5/FP RNA can be competed by both unlabeled E5/FP and E5/FPD RNA. Each reaction contained $0.4 \mu \mathrm{g}$ of recombinant hnRNP Al and $7 \mu \mathrm{g}$ of BSA. (Lane 1) No competitor was added. Molar excess (7.5-, 15-, and 30-fold) of unlabeled E5/FP RNA (lanes 2-4) or unlabeled E5/FPD RNA (lanes 5-7) was added together with labeled E5/FP RNA. Protein standards are as in Fig. 1. 
amount of $35-\mathrm{kD}$ protein (Fig. 6A, lane 5). This band was only visible after long exposure times (data not shown), perhaps reflecting the fact that hnRNP Al levels in $\$ 100$ extracts are much lower than in nuclear extracts (Mayeda et al. 1993). When E5/FPD RNA was crosslinked in HeLa cell nuclear extract, anti-hnRNP Al mAb also precipitated the labeled $35-\mathrm{kD}$ protein (Fig. 6A, lane 6), suggesting that although hnRNP Al binds to the FP element, it also binds to other exon 5 sequences.

To address further the binding specificity of hnRNP $A 1$, purified recombinant hnRNP Al protein was used in UV cross-linking experiments. Recombinant hnRNP A1 was cross-linked to E5/FP RNA, either in the presence or absence of ATP and $\mathrm{Mg}^{2+}$ (Fig. 6B, lanes 2,3). Recombinant hnRNP A1 protein also cross-linked to E5/FPD RNA under similar conditions (Fig. 6B, lanes 4,5), although with slightly lower efficiency. This was confirmed further by a competition assay in which the crosslinking of recombinant hnRNP Al to labeled E5/FP RNA was measured in the presence of increasing amounts of unlabeled E5/FP RNA or E5/FPD RNA. Both RNA sequences decreased hnRNP Al binding to the labeled E5/FP RNA, although unlabeled E5/FP RNA competed slightly better (Fig. 6C). Compared with SF2/ASF, hnRNP Al binds to the FP element in the bGH terminal exon with substantially lower specificity. The observed selectivity ratio for binding to the two RNAs was approximately 2 .

\section{Discussion}

In this study we have demonstrated that the general splicing factor SF2/ASF binds specifically and directly to the FP element, a purine-rich ESE, in the bGH $3^{\prime}$ terminal exon. This specific protein-RNA interaction results in activation of splicing of the preceding bGH intron $\mathrm{D}$. However, the related SR protein SC35 does not have the same effect on bGH intron D splicing as SF2/ASF. Furthermore, we have shown that hnRNP Al counteracts the positive influence of SF2/ASF on the splicing of bGH intron D.

Expression of a transiently transfected bGH gene in several different cell lines, including COS $1, \mathrm{HeLa}, \mathrm{NIH}$ $3 \mathrm{~T} 3$, and $\mathrm{CHO}$, consistently results in the presence of $\sim 5 \%$ intron D-containing bGH mRNA species on polysomes (R.K. Hampson, unpubl.). The range of cell types producing this low level of alternatively spliced mRNA argues for the involvement of constitutive, rather than tissue-specific, splicing factor(s). Immunoprecipitation with anti-SF2/ASF mAb showed that the $35-\mathrm{kD}$ protein factor(s) includes the general splicing factor SF2/ASF. However, we were not able to address whether SF2/ASF is the only $35-\mathrm{kD}$ protein in HeLa cell nuclear extracts that binds specifically to the FP element, because antiSF2/ASF mAb did not quantitatively precipitate either the 35-kD labeled band or purified SF2/ASF. Anti-SF2/ ASF mAb immunoprecipitated SF2/ASF in nuclear extract or in purified form with similar, albeit low, efficiency, suggesting that most of the $35-\mathrm{kD}$ protein that binds to the FP element in HeLa cell nuclear extract is
SF2/ASF. However, the immunoprecipitation experiments with anti-hnRNP Al mAb suggest that other 35$\mathrm{kD}$ proteins may also bind to the FP element with undetermined levels of specificity.

Experiments with recombinant SF2/ASF confirmed that this factor is responsible for stimulating $\mathrm{bGH}$ intron D splicing by binding specifically and directly to the ESE. In contrast, recombinant SC35 that was highly active in S100 complementation assays with $\beta$-globin and adenovirus pre-mRNA substrates was unable to bind directly to the ESE or to activate bGH intron D splicing. These results demonstrate that the observed effects of SF2/ASF on bGH intron D splicing are not a simple reflection of general splicing stimulation.

Although previous results indicated that the general splicing factor SF2/ASF has general RNA-binding activity [e.g., binding to sense and antisense pre-mRNA or mRNA (Krainer et al. 1990a; data not shown)], these experiments did not exclude the existence of high-affinity sequence-specific binding sites. Our present results clearly indicate that SF2/ASF has a higher binding affinity for the FP element over other bGH exon 5 sequences, with a selectivity ratio $>10$. This binding preference is significant because only the FP element, and not the other downstream exon 5 sequences, is capable of activating splicing of the upstream bGH intron $D$. These results strongly suggest that SF2/ASF promotes the splicing of bGH intron $D$ through specific interaction with the FP element in the downstream exon 5. Although we do not as yet know the precise sequences within the 115-nucleotide FP element that are recognized by SF2/ASF, recent transfection studies have shown that purine-rich elements within the FP sequence function as cis-acting signals for splicing activation of the upstream bGH intron $\mathrm{D}$, which contains a weak $5^{\prime}$ splice site (Dirksen et al. 1994). Purine-rich exon sequences have also been reported to be involved in alternative or constitutive splicing of several other pre-mRNAs (Watakabe et al. 1993; Xu et al. 1993). It will be of interest to determine whether the general splicing factor SF2/ASF and perhaps other SR proteins bind specifically to some or all purine-rich elements to promote splicing of weak introns, that is, introns with suboptimal splicing signals. On the other hand, some constitutively spliced introns with strong $5^{\prime}$ and $3^{\prime}$ splice sites and branch sites would not be expected to require exonic enhancer elements for efficient splicing. Such pre-mRNAs, for example, $\beta$-globin, also require SF2/ASF (or other SR proteins) for splicing in vitro (Krainer et al. 1990a); and in these cases, it remains to be determined whether high-affinity binding sites for SF2/ASF are also required and whether they are located in exons or introns.

In vitro splicing of an HIV tat pre-mRNA is strongly dependent on an excess of SF2/ASF (Krainer et al. 1990a). Examination of the nucleotide sequence of the $3^{\prime}$ terminal exon of this substrate reveals long purine-rich segments. This suggests that activation of otherwise weak tat and bGH splicing by excess SF2/ASF may have a similar mechanistic basis. Recently, it was shown that in the presence of excess competitor RNA, SF2/ASF, but 
not SC35, can form a commitment complex with the same tat pre-mRNA in vitro, whereas SC35 commits $\beta$-globin pre-mRNA more efficiently than SF2/ASF (Fu 1993). The formation of a specific commitment complex with an SR protein in vitro is consistent with the presence of a high-affinity binding site for that SR protein.

hnRNP Al counteracts SF2/ASF to promote the use of distal alternative 5 ' splice sites and also to promote certain alternative exon skipping events (Mayeda and Krainer 1992; Mayeda et al. 1993). These effects are concentration dependent with respect to both SF2/ASF and hnRNP Al. In this study we demonstrate that hnRNP Al also counteracts SF2/ASF to promote alternative intron retention of bGH pre-mRNA. Thus, it appears that $\mathrm{hnRNP} A 1$ is able to antagonize SF2/ASF in a variety of alternative pre-mRNA splicing events, although this is not the case for the activity of SF2/ASF that stimulates proximal alternative 3' splice sites (Fu et al. 1992).

Compared with SF2/ASF, hnRNP A1 binds with low specificity to the FP element, with at least fivefold lower selectivity between two exon 5 RNA fragments containing or lacking the ESE. This observation does not exclude the possibility that hnRNP Al recognizes certain sequences with high affinity, both in bGH and in other pre-mRNAs. The opposing effects of hnRNP and SF2/ ASF on the splicing of bGH intron D could be explained by competition between the two proteins for overlapping binding sites. Alternatively, hnRNP Al may block protein-protein or protein-RNA interactions between SF2/ ASF and other splicing factors required to recognize the weak $5^{\prime}$ splice site, such as U1 snRNP.

Previous experiments showed that specific competitor RNA containing the FP element inhibited splicing of bGH intron $\mathrm{D}$ but not of a control adenovirus intron. These results suggested that splicing of bGH intron $D$ is especially sensitive to changes in the levels of the putative splicing factor binding to the FP element (Sun et al. 1993). We found that addition of hnRNP Al and SF2/ ASF proteins in similar amounts to those used to affect bGH alternative splicing had no detectable effect on splicing of adenovirus and $\beta$-globin introns. Moreover, the stimulatory effect of SF2/ASF addition on intron D removal was demonstrated in HeLa cell nuclear extract, where SF2/ASF is already present. Therefore, SF2/ASF appears to be limiting for bGH intron D splicing in vitro, as was shown previously to be the case for an HIV tat pre-mRNA that is naturally spliced inefficiently, but not for constitutively spliced pre-mRNAs (Krainer et al. 1990a). Natural fluctuations of the relative levels of SF2/ ASF and hnRNP Al may serve to regulate the extent to which an intron is retained or spliced in different tissues or developmental stages.

The bGH intron D has a weak $5^{\prime}$ splice site which, when mutated to the mammalian $5^{\prime}$ splice site consensus sequence, converts it into a constitutively spliced intron in vivo, independent of the presence of the FP element in the downstream exon (Dirksen et al. 1994). This is consistent with the notion that the selection of suboptimal splice sites is more sensitive to the fluctuation of certain constitutive splicing factors-in this case, the general splicing factor SF2/ASF (Krainer et al. 1990b; Dominski and Kole 1992; Mayeda et al. 1993). Moreover, the possible role of the FP element in compensating for a weak $5^{\prime}$ splice site suggests that the mechanism of action of SF2/ASF in alternative intron retention of bGH pre-mRNA may be related to the mechanism of alternative $5^{\prime}$ splice site selection of internal exons.

The exon definition model proposes that the splicing machinery first scans and defines the downstream exon, as an obligatory step in the identification of the upstream intron (Robberson et al. 1990; Talerico and Berget 1990). Exon definition is proposed to occur through interactions between factors bound at the 3 ' splice site of the intron being removed and at the downstream $5^{\prime}$ splice site (Robberson et al. 1990). Interactions defining a preprotachykinin alternative exon have been shown to include binding of $U 2 \mathrm{AF}^{65}$ at the $3^{\prime}$ splice site and of $\mathrm{U} 1$ snRNP at the downstream $5^{\prime}$ splice site, via at least one other protein factor bridging across the exon (Hoffman and Grabowski 1992). The polyadenylation signal has been proposed to fulfill a role analogous to that of a functional downstream 5' splice site in the case of 3'-terminal exons (Niwa and Berget 1991). Alternatively, internal exon sequences with limited complementarity to U1 snRNA, which influence splicing of the upstream intron, may function as the exon definition signals for the 3'terminal exons (Wassarman and Steitz 1993; Watakabe et al. 1993).

Our experiments suggest an additional mechanism, in which a protein factor mediating interaction between $\mathrm{U} 2 \mathrm{AF}$ and U1 snRNP may bind directly to an element within the $3^{\prime}$-terminal exon and thus help to define the last exon. We showed that SF2/ASF activates splicing of the upstream bGH intron $\mathrm{D}$ through specific interaction with a downstream exon element, suggesting that one or more SR proteins, in this case, SF2/ASF, may be responsible for the recognition of 3 '-terminal exons. Interestingly, the third component required to bridge the interaction between U2AF and U1 snRNP across the preprotachykinin exon 4, is absent in HeLa cell S100 extract (Hoffman and Grabowski 1992); hence, it may be one of the SR proteins. Interactions between SF2/ASF and U1 snRNP, which promote the binding of U1 snRNP to $5^{\prime}$ splice sites, have been demonstrated recently (Eperon et al. 1993). SC35, another member of the SR protein family, has been found to be capable of mediating interactions between U1 and U2 snRNPs at the 3' splice site (Fu and Maniatis 1992). Therefore, one of the functions of SR proteins may be to interact with $\mathrm{U} 2 \mathrm{AF}$ and $\mathrm{U} 1$ and $\mathrm{U} 2$ snRNPs during exon definition and splice site selection.

The mechanism of regulation of Drosophila dsx female-specific alternative splicing also involves specific binding of proteins to a downstream exon element, and interestingly, the splicing enhancer complex that forms at this site includes SR proteins (Tian and Maniatis 1993). The $d s x$ exonic enhancer, which is not purinerich, consists of six 13-nucleotide repeats not found in other genes. It is recognized by specific regulatory proteins encoded by tra and tra2, which in turn recruit SR proteins to form a commitment complex. In contrast, in 
the bGH system we have shown that there is direct contact between at least one SR protein, SF2/ASF, and the bGH exonic enhancer, which includes purine-rich segments. In addition, the $d s x$ splicing commitment complex forms more efficiently with SC35 than with SF2/ ASF (Tian and Maniatis 1993), whereas bGH intron D splicing is stimulated by SF2/ASF but not by SC35. However, once the respective splicing enhancer complexes are assembled, the interactions mediated by bound SR proteins, which lead to splicing activation, may be very similar in both cases. The idea that exonic enhancers operate by similar mechanisms, aside from their use of specialized regulatory proteins in certain instances, is supported further by the observation that the purine-rich splicing enhancer from the murine IgM last exon can functionally replace the Drosophila $d s x$ enhancer, leading to activation of the upstream intron in a Tra/Tra2-independent manner (Watakabe et al. 1993). The similar sequence composition and downstream exon location of vertebrate purine-rich splicing enhancers, including the bGH FP element, was noted previously (Watakabe et al. 1993). This similarity suggests that direct binding by SF2/ASF (and/or other SR proteins), counteracted by hnRNP Al, may be a general mechanism for regulating alternative splicing of pre-mRNAs that contain purine-rich splicing enhancers.

\section{Materials and methods}

\section{Plasmid constructions}

The plasmid E5/FP contains a 115-nucleotide FspI-PvuII restriction fragment from bGH exon 5, derived from plasmid pbGH-4D5 (Sun et al. 1993), which was subcloned into the EcoRI-SmaI sites of pBS-M13 +. The plasmid E5/FPD contains a 135-nucleotide SmaI-EcoRI restriction fragment from the bGH exon 5, derived from plasmid pbGH-4D5/FPD (Sun et al. 1993), which was subcloned into the EcoRI-SmaI sites of pBS$\mathrm{M} 13+$.

\section{Splicing factors}

Human SF2/ASF $(0.2 \mathrm{mg} / \mathrm{ml})$ was purified from HeLa cell $\mathrm{nu}$ clear extract as described (Mayeda et al. 1993). This highly purified fraction is devoid of SC35 and other SR proteins (data not shown). Human recombinant hnRNP Al $(0.2 \mathrm{mg} / \mathrm{ml})$ was expressed in Escherichia coli and purified as described (Mayeda and Krainer 1992). Recombinant human SF2/ASF $(0.65 \mathrm{mg} / \mathrm{ml})$ was expressed in E. coli, as described (Càceres and Krainer 1993|, and purified from inclusion bodies by chromatography on Mono S (Pharmacia) in $6 \mathrm{~m}$ urea, followed by renaturation. Recombinant SC35 $(0.13 \mathrm{mg} / \mathrm{ml})$ purified from baculovirus-infected insect cells was a generous gift from T. Maniatis (Harvard University, Cambridge, MA). Protein concentrations were determined by the dye-binding assay (Bio-Rad) with BSA as a standard.

\section{Splicing reactions and UV cross-linking assays}

HeLa cell nuclear extract and S100 extract were prepared essentially as described (Dignam et al. 1983). All splicing reactions were performed as described (Sun et al. 1993), except that $4 \mu$ l of nuclear extract was used in each reaction either alone or together with purified splicing factors as indicated in Figures 3 and 4.

The plasmids E5/FP and E5/FPD were linearized with BamHI, transcribed in vitro with T7 RNA polymerase, and used in UV cross-linking assays as described (Sun et al. 1993), except that the RNase A-treated cross-linked products were analyzed by $10 \%$ SDS-PAGE.

\section{UV cross-linking/immunoprecipitation assays}

The anti-hnRNP Al mAb 4B10 was a generous gift from Drs. G. Dreyfuss and S. Piñol-Roma (University of Pennsylvania, Philadelphia). The anti-SF2/ASF $\mathrm{mAb}$ and a control monoclonal antibody against a bacterial protein will be described elsewhere; the anti-SF2/ASF mAb does not cross-react with other SR proteins by Western blotting (A. Hanamura and A.R. Krainer, in prep.). Anti-hnRNP Al mAb in ascites fluid $(2.5 \mu \mathrm{l})$ or anti-SF2/ ASF mAb or control monoclonal antibody culture supernatants (300 $\mu l)$ were used in each immunoprecipitation reaction. AntibGH mAb (Salata et al. 1992) (2 mg/ml) was used in a preclearing step. Radiolabeled E5/FP or E5/FPD RNA was first crosslinked in either HeLa cell nuclear extract or S100 extract and treated with RNase A as described (Sun et al. 1993). Each reaction was precleared with $5 \mu \mathrm{l}$ of bGH mAb and $50 \mu \mathrm{l}$ of $50 \%$ protein A agarose in $500 \mu \mathrm{l}$ of IP buffer $(50 \mathrm{mM}$ Tris at $\mathrm{pH} 8.0$, $150 \mathrm{~mm} \mathrm{NaCl}, 0.05 \% \mathrm{NP}-40$ ) and mixed for $1 \mathrm{hr}$ at $4^{\circ} \mathrm{C}$. At the same time, each antibody was prebound to $50 \mu$ l of protein A-agarose $(50 \%)$ in $500 \mu \mathrm{l}$ of IP buffer for $1 \mathrm{hr}$ and washed twice with $1 \mathrm{ml}$ of IP buffer for $15 \mathrm{~min}$ at $4^{\circ} \mathrm{C}$. After spinning in a microcentrifuge for $30 \mathrm{~min}$ at $4^{\circ} \mathrm{C}$, the supernatant was incubated further with each monoclonal antibody immobilized on protein A-agarose. The immunoprecipitation reactions were mixed at $4^{\circ} \mathrm{C}$ overnight. After six washes with $1 \mathrm{ml}$ of IP buffer at $4^{\circ} \mathrm{C}$, each sample was eluted in $40 \mu \mathrm{l}$ of $1 \times$ sample buffer $162.5 \mathrm{~mm}$ Tris at $\mathrm{pH} 6.8,2 \%$ SDS, $10 \%$ glycerol, $5 \% \beta$-mercaptoethanol) at $85^{\circ} \mathrm{C}$ for $10 \mathrm{~min}$ and analyzed by $10 \%$ SDS-PAGE.

\section{Acknowledgments}

We thank Drs. Gideon Dreyfuss and Serafin Piñl-Roma for antihnRNP Al monoclonal antibody, and Drs. Xiang-Dong Fu and Tom Maniatis for recombinant SC35 protein. We are grateful to Wessel P. Dirksen for critical reading of the manuscript and helpful suggestions throughout the course of this work. A.M. was supported by a long-term fellowship from the International Human Frontier Science Program Organization. A.R.K is a Pew Scholar in the Biomedical Sciences. This work was supported by National Institutes of Health grants (DK-32770) to F.M.R. and (GM-42699) to A.R.K.

The publication costs of this article were defrayed in part by payment of page charges. This article must therefore be hereby marked "advertisement" in accordance with 18 USC section 1734 solely to indicate this fact.

\section{References}

Boggs, R.T., T. Gregor, S. Idriss, M.J. Belote, and M. McKeown. 1987. Regulation of sexual differentiation in D. melanogaster via alternative splicing of RNA from the transformer gene. Cell 50: 739-747.

Càceres, J.F. and A.R. Krainer. 1993. Functional analysis of premRNA splicing factor SF2/ASF structural domains. EMBO J. 12: 4715-4726. 
Cooke, N.E., J. Ray, J.G. Emery, and S.A. Liebhaber. 1988. Two distinct species of human growth hormone-variant mRNA in the human placenta predict the expression of novel growth growth proteins. J. Biol. Chem. 263: 9001-9006.

Cooper, T.A. 1992. In vitro splicing of cardiac troponin T precursors. J. Biol. Chem. 267: 5330-5338.

Cooper, T.A. and C.P. Ordahl. 1989. Nucleotide substitutions within the cardiac troponin $\mathrm{T}$ alternative exon disrupt premRNA alternative splicing. Nucleic Acids Res. 17: 79057921.

Cote, G.J., D.T. Stolow, S. Peleg, S.M. Berget, and R.F. Gagel. 1992. Identification of exon sequences and an exon binding protein involved in alternative RNA splicing of calcitonin/ CGRP. Nucleic Acids Res. 20: 2361-2366.

Dignam, J.D., R.M. Lebovitz, and R.G. Roeder. 1983. Accurate transcription initiation by RNA polymerase II in a soluble extract from isolated mammalian nuclei. Nucleic Acids Res. 11: 1475-1489.

Dirksen, W.P., R.K. Hampson, Q. Sun, and F.M. Rottman. 1994. A purine-rich exon sequence influences alternate splicing of bovine growth hormone pre-mRNA. J. Biol. Chem. (in press).

Dominski, Z. and R. Kole. 1992. Cooperation of pre-mRNA sequence elements in splice site selection. Mol. Cell. Biol. 12: $2108-2114$

Eperon, I.C., D.C. Ireland, R.A. Smith, A. Mayeda, and A.R. Krainer. 1993. Pathways for selection of 5 ' splice sites by U1 snRNP and SF2/ASF. EMBO I. 12: 3607-3617.

Fu, X.-D. 1993. Specific commitment of different pre-mRNAs to splicing by single SR proteins. Nature 365: 82-85.

$\mathrm{Fu}, \mathrm{X}$.-D. and T. Maniatis. 1992. The 35-kDa mammalian splicing factor SC35 mediates specific interactions between U1 and U2 small nuclear ribonucleoprotein particles at the 3' splice site. Proc. Natl. Acad. Sci. 89: 1725-1729.

Fu, X.-D., A. Mayeda, T. Maniatis, and A.R. Krainer. 1992. General splicing factors SF2 and SC35 have equivalent activities in vitro, and both affect alternative $5^{\prime}$ and $3^{\prime}$ splice site selection. Proc. Natl. Acad. Sci. 89: 11224-11228.

Ge, H. and J.L. Manley. 1990. A protein factor, ASF, controls cell-specific alternative splicing of SV40 early pre-mRNA in vitro. Cell 62: 25-34.

Hampson, R.K. and F.M. Rottman. 1987. Alternative processing of bovine growth hormone mRNA: Nonsplicing of the final intron predicts a high molecular weight variant of bovine growth hormone. Proc. Natl. Acad. Sci. 84: 2673-2677.

Hampson, R.K., L. LaFollette, and F.M. Rottman. 1989. Alternative processing of bovine growth hormone mRNA is influenced by downstream exon sequences. Mol. Cell. Biol. 9: $1604-1610$.

Hedley, M.L. and T. Maniatis. 1991. Sex-specific splicing and polyadenylation of dsx pre-mRNA requires a sequence that binds specifically to Tra-2 protein in vitro. Cell 65: $579-586$.

Helfman, D.M., W.M. Ricci, and L.A. Finn. 1988. Alternative splicing of tropomyosin pre-mRNAs in vitro and in vivo. Genes \& Dev. 2: 1627-1638.

Hoffman, B.E. and P.J. Grabowski. 1992. U1 snRNP targets an essential splicing factor, U2AF, to the $3^{\prime}$ splice site by a network of interactions spanning the exon. Genes \& Dev. 6: 2554-2568.

Hoshijima, K., K. Inoue, I. Higuchi, H. Sakamoto, and Y. Shimura. 1991. Control of doublesex alternative splicing by transformer and transformer-2 in Drosophila. Science 252: 833-835.

Kakizuka, A., T. Ingi, T. Murai, and S. Nakanishi. 1990. A set of U1 snRNA-complementary sequences involved in governing alternative RNA splicing of the kininogen genes. I. Biol. Chem. 265: 10102-10108.
Katz, R.A. and A.M. Skalka. 1990. Control of retroviral RNA splicing through maintenance of suboptimal processing signals. Mol. Cell. Biol. 10: 696-704.

Kim, Y.-J., P. Zuo, J.L. Manley, and B.S. Baker. 1992. A Drosophila RNA binding protein RBPl is localized to transcriptionally active sites of chromosomes and shows a functional similarity to human splicing factor ASF/SF2. Genes \& Dev. 6: $2569-2579$.

Krainer, A.R., G.C. Conway, and D. Kozak. 1990a. Purification and characterization of pre-mRNA splicing factor SF2 from HeLa cells. Genes \& Dev. 4: 1158-1171.

- 1990b. The essential pre-mRNA splicing factor SF2 influences $5^{\prime}$ splice site selection by activating proximal sites. Cell 62: 35-42.

Libri, D., A. Piseri, and M.Y. Fiszman. 1991. Tissue-specific splicing of $\beta$-tropomyosin gene: Dependence on an RNA secondary structure. Science 252: 1842-1845.

Mardon, H.J., G. Sebastio, and F.E. Barralle. 1987. A role for exon sequence in alternative splicing of the human fibronectin gene. Nucleic Acids Res. 15: 7725-7733.

Mayeda, A. and A.R. Krainer. 1992. Regulation of alternative pre-mRNA splicing by hnRNP Al and splicing factor SF2. Cell 68: 365-375.

Mayeda, A., A.M. Zahler, A.R. Krainer, and M.B. Roth. 1992. Two members of a conserved family of nuclear phosphoproteins are involved in pre-mRNA splicing. Proc. Natl. Acad. Sci. 89: 1301-1304.

Mayeda, A., D.M. Helfman, and A.R. Krainer. 1993. Modulation of exon skipping and inclusion by heterogeneous nuclear ribonucleoprotein $\mathrm{A} 1$ and pre-mRNA splicing factor SF2/ ASF. Mol. Cell. Biol. 13: 2993-3001.

Nagoshi, R.N. and B.S. Baker. 1990. Regulation of sex specific RNA splicing at the Drosophila doublesex gene: Cis-acting mutations in exon sequences alter sex-specific RNA splicing patterns. Genes \& Dev. 4: 89-97.

Niwa, M. and S.M. Berget. 1991. Mutation of the AAUAAA polyadenylation signal depresses in vitro splicing of proximal but not distal introns. Genes \& Dev. 5: 2086-2095.

Reed, R. and T. Maniatis. 1986. A role for exon sequences and splice-site proximity in splice-site selection. Cell 46: 681690.

Robberson, B.L., G.J. Cote, and S.M. Berget. 1990. Exon definition may facilitate splice site selection in RNAs with multiple exons. Mol. Cell. Biol. 10: 84-94.

Salata, R.A., I.J. Malhotra, R.K. Hampson, D.F. Ayers, C.-S. Tomich, and F.M. Rottman. 1992. Application of an immune-tolerizing procedure to generate monoclonal antibodies specific to an alternate protein isoform of bovine growth hormone. Anal. Biochem. 207: 142-149.

Siebel, C.W., L.D. Fresco, and D.C. Rio. 1992. The mechanism of somatic inhibition of Drosophila P-element pre-mRNA splicing: Multiprotein complexes at an exon pseudo-5' splice site control Ul snRNP binding. Genes \& Dev. 6: 1386-1401.

Sun, Q., R.K. Hampson, and F.M. Rottman. 1993. In vitro analysis of bovine growth hormone hormone pre-mRNA alternative splicing: Exon sequences and trans-acting factor(s). J. Biol. Chem. 268: 15659-15666.

Talerico, M. and S.M. Berget. 1990. Effect of 5' splice site mutation on splicing of the preceding intron. Mol. Cell. Biol. 10: 6299-6305.

Tian, M. and T. Maniatis. 1992. Positive control of pre-mRNA splicing in vitro. Science 256: 237-240.

-1993. A splicing enhancer complex controls alternative splicing of doublesex pre-mRNA. Cell 74: 105-114.

Valcarcel, J., R. Singh, P.D. Zamore, and M.R. Green. 1993. The protein sex-lethal antagonizes the splicing factor U2AF to 
Sun et al.

regulate alternative splicing of transformer pre-mRNA. $\mathrm{Na}$ ture 362: 171-175.

Wassarman, K.M. and J.A. Steitz. 1993. Association with terminal exons in pre-mRNAs: A new role for U1 snRNP? Genes \& Dev. 7: 647-659.

Watakabe, A., K. Tanaka, and Y. Shimura. 1993. The role of exon sequences in splice site selection. Genes \& Dev. 7: 407-418.

$\mathrm{Xu}, \mathrm{R} ., \mathrm{J}$. Teng, and T.A. Cooper. 1993. The cardiac troponin T alternative exon controls a novel purine-rich positive splicing element. Mol. Cell. Biol. 13: 3660-3647.

Zahler, A.M., W.S. Lane, J.A. Stolk, and M.B. Roth. 1992. SR proteins: A conserved family of pre-mRNA splicing factors. Genes \& Dev. 6: 837-847.

Zahler, A.M., K.M. Neugebauer, W.S. Lane, and M.B. Roth. 1993. Distinct functions of SR proteins in alternative premRNA splicing. Science 260: 219-222. 


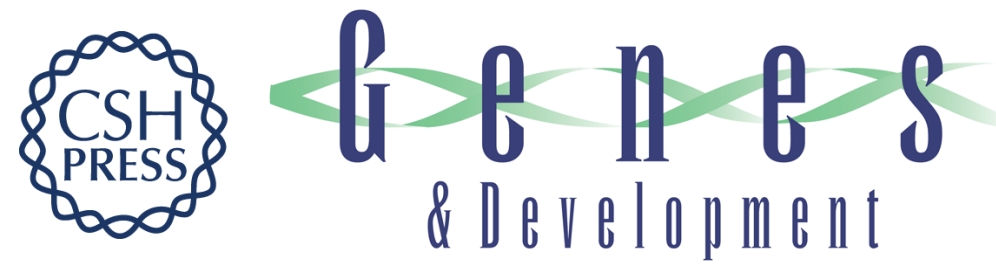

\section{General splicing factor SF2/ASF promotes alternative splicing by binding to an exonic splicing enhancer.}

Q Sun, A Mayeda, R K Hampson, et al.

Genes Dev. 1993, 7:

Access the most recent version at doi:10.1101/gad.7.12b.2598

References This article cites 44 articles, 28 of which can be accessed free at:

http://genesdev.cshlp.org/content/7/12b/2598.full.html\#ref-list-1

License

Email Alerting

Service

Receive free email alerts when new articles cite this article - sign up in the box at the top right corner of the article or click here.

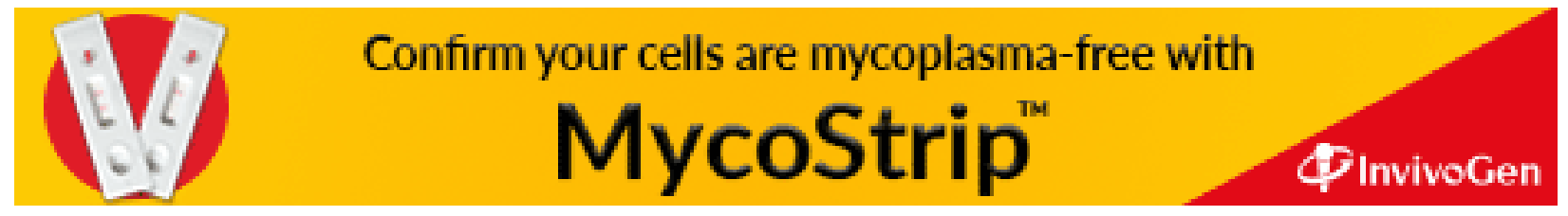

